\title{
Phosphate-induced-1 gene from Eucalyptus (EgPHI-1) enhances osmotic stress tolerance in transgenic tobacco
}

\author{
A.O. Sousa, E.T.C.M. Assis, C.P. Pirovani, F.C. Alvim and M.G.C. Costa \\ Centro de Biotecnologia e Genética, Departamento de Ciências Biológicas, \\ Universidade Estadual de Santa Cruz, Ilhéus, BA, Brasil \\ Corresponding author: M.G.C. Costa \\ E-mail: marciogc.costa@gmail.com
}

Genet. Mol. Res. 13 (1): 1579-1588 (2014)

Received January 21, 2013

Accepted June 29, 2013

Published March 12, 2014

DOI http://dx.doi.org/10.4238/2014.March.12.10

\begin{abstract}
Environmental stresses such as drought, freezing, and high salinity induce osmotic stress in plant cells. The plant response to osmotic stress involves a number of physiological and developmental changes, which are made possible, in part, by the modulation of the expression of specific genes. Phosphate-induced-1 gene (PHI-1) was first isolated from phosphate-treated phosphate-starved tobacco cell cultures as a stress-inducible gene, which is presumably related to intracellular $\mathrm{pH}$ maintenance; however, the role of the PHI-1 gene product has not yet been clarified. A gene encoding a predicted protein with high similarity to tobacco PHI-1, named EgPHI-1, was previously identified in Eucalyptus by comparative transcriptome analysis of xylem cells from species of contrasting phenotypes for wood quality and growth traits. Here, we show that the overexpression of EgPHI-1 in transgenic tobacco enhances tolerance to osmotic stress. In comparison with wild-type plants, EgPHI- 1 transgenic plants showed a significant increase in root length and biomass dry weight under $\mathrm{NaCl}$, , polyethylene glycol, and mannitol-induced osmotic stresses. The enhanced stress tolerance of transgenic plants was correlated
\end{abstract}


with increased endogenous protein levels of the molecular chaperone binding protein $\mathrm{BiP}$, which in turn was correlated with the EgPHI-I expression level in the different transgenic lines. These results provide evidence about the involvement of EgPHI-1 in osmotic stress tolerance via modulation of $\mathrm{BiP}$ expression, and pave the way for its future use as a candidate gene for engineering tolerance to environmental stresses in crop plants.

Key words: Intracellular pH; Phosphorylation; Abiotic stress; Chaperone; Endoplasmic reticulum; Cell death

\section{INTRODUCTION}

Environmental stresses, such as drought, freezing, and high salinity, are often interconnected since they may induce similar cellular damage that is primarily manifested as osmotic stress. As environmental stress conditions seriously affect crop production in many parts of the world, the molecular and cellular processes underlying the acclimation of plants to osmotic stress have attracted much research interest recently (Zhu, 2002; Mahajan and Tuteja, 2005; Vásquez-Robinet et al., 2010). To cope with osmotic stress, plants have developed a number of physiological and developmental mechanisms, including alteration of life cycle, inhibition of shoot growth and enhancement of root growth, adjustment of ion transport, carbon metabolism, and the synthesis of compatible solutes (Xiong and Zhu, 2002). Many of these changes are made possible by modulations of the expression of specific genes, which provides an important approach for engineering tolerance in plants (Brinker et al., 2010).

A complex apparatus of signaling factors is required for the transcription of stressinducible genes. These factors perceive environmental variations and transduce these signals through cells. Changes in intracellular $\mathrm{pH}$ are one of the first indicators of osmotic stress perceived by cells (Netting, 2000). Metabolism, transport, and signaling are homeostatic processes that are fully dependent on the regulation of intracellular $\mathrm{pH}$ in plant cells (Sakano, 1998). For example, the phosphate-induced-1 gene (PHI-1), which was first identified in cell cultures of tobacco (Sano et al., 1999), has attracted considerable interest. Despite its sequence similarity to the EXORDIUM (EXO) gene of Arabidopsis, PHI-1 and EXO do not show similarities to any gene with known function. Tobacco $P H I-1$ was initially presumed to play a role in phosphate-induced cell cycle re-entry (Sano et al., 1999). Subsequently, the PHI1 gene product was inferred to play a role in alleviating changes of intracellular $\mathrm{pH}$ caused by stress conditions in cells (Sano and Nagata, 2002). However, the exact role of the PHI-1 gene product has not yet been clarified.

Comparative analysis between the xylem transcriptomes of Eucalyptus grandis and Eucaplytus globulus, using high-density microarrays, led to the identification of a differentially expressed gene encoding a predicted protein with high similarity to tobacco PHI-1 (Pasquali et al., 2005). This gene, named EgPHI-1, was expressed 7.5 times more in E. globulus than in E. grandis (Pasquali et al., 2005), two Eucalyptus species with contrasting growth and wood characteristics. E. grandis is a species native to tropical and subtropical regions that exhibits rapid growth, but produces a low-density wood. On the other hand, E. globulus is a species from temperate climates that produces a high-density wood, but exhibits slow growth 
when cultivated in tropical regions (Eldridge et al., 1993; Bernard, 2003; Myburg et al., 2007). Considering the economic and ecological relevance of the genus Eucalyptus, understanding the cellular function of stress-inducible genes is of central interest for biotechnological applications in forestry.

In this study, we investigated the relationship between EgPHI-1 and osmotic stress. Transgenic tobacco plants overexpressing EgPHI-1 were generated and subjected to osmotic stress induced by $\mathrm{NaCl}$, mannitol, and polyethylene glycol (PEG). The results indicated that EgPHI-1 enhances osmotic stress tolerance of transgenic plants through a mechanism that involves the modulation of the expression of the molecular chaperone binding protein, BiP, an important component of the endoplasmic reticulum (ER) that protects the cell against stresses.

\section{MATERIAL AND METHODS}

\section{Plant material}

The EgPHI-1-coding sequence, which was identified in a xylem cDNA library of $E$. globulus, was released from the pSPORT1 cloning vector by SalI and NotI digestion. The purified $\sim 1.2-\mathrm{kb}$ fragment was subcloned in the sense orientation between the promoter and terminator sequences of the cauliflower mosaic virus (CaMV) $35 \mathrm{~S}$ of the $\mathrm{pUC1} 18$ vector using the XhoI and NotI restriction sites. The resulting 35S::EgPHI-1 construct was released from pUC118 by BamHI/HindIII double-digestion and inserted into the same restriction sites of the binary vector pCAMBIA 2301 (CAMBIA, Australia), which also contains the neomycin phosphotransferase (nptII) and $\beta$-glucuronidase (uidA) genes under the control of the CaMV $35 \mathrm{~S}$ promoter. The plasmid construct was introduced into Agrobacterium tumefaciens EHA105 by direct DNA uptake. The construction was transferred to wild-type (WT) tobacco (Nicotiana tabacum cv. Havana) by A. tumefaciens-mediated genetic transformation, as previously described (Horsch et al., 1985). Three transgenic lines (L1, L2, and L3), representing distinct transformation events, were selected on appropriate media by their resistance to kanamycin, positive reaction in GUS histochemical assays (Jefferson, 1989), polymerase chain reaction (PCR) amplification of $n p t I I$ and $u i d \mathrm{~A}$, and by reverse transcription-quantitative real-time PCR (RT-qPCR) (Sousa AO, Assis ETCM, Silva DC, et al., unpublished results). Seeds from WT and kanamycin-resistant $T_{1}$ transgenic plants used in the osmotic stress experiments were derived from plants grown in a greenhouse under identical conditions.

\section{Osmotic stress experiments}

Seeds from WT and transgenic plants were sterilized and plated on MS medium (Murashige and Skoog, 1962) solidified with $0.7 \%$ agar. After 2 days in a cold room $\left(4^{\circ} \mathrm{C}\right)$, the plates were transferred to a growth chamber with a long day light regime (16 h day, 140 $\mu \mathrm{mol} \cdot \mathrm{m}^{-2} \cdot \mathrm{s}^{-1}, 27^{\circ} \pm 2^{\circ} \mathrm{C} ; 8 \mathrm{~h}$ night, $27^{\circ} \pm 2^{\circ} \mathrm{C}$ ), and grown in a randomized manner over 15 days. After this period, nine plants of similar size from each of the WT and transgenic lines were transferred to MS medium supplemented with the stress inductors, $\mathrm{NaCl}$, mannitol, or PEG, in concentrations sufficient to impose a water potential of -1 MPa. The WT and transgenic plants were transferred to MS medium without stress inductors in the control treatment. The plants were maintained in a growth chamber over the next 25 days under the same con- 
ditions described above. At the end of this period, phenotypic traits, including leaf wilting, chlorosis, and necrotic lesions, were analyzed, along with the root length and the biomass dry weight of individual plants. To determine the biomass dry weight, plants were kept in an air-circulation stove at $75^{\circ} \mathrm{C}$ until reaching a constant weight. All treatments were performed in triplicate.

\section{Protein extraction and Western blot analysis}

Leaves of control and stressed WT and transgenic plants were used for BiP immunoblot analyses. These plants were grown for 60 days in a greenhouse under identical normal conditions. Lyophilized leaves were macerated in liquid nitrogen and $1 \%(\mathrm{w} / \mathrm{w})$ polyvinylpyrrolidone by the phenol/SDS method (Pirovani et al., 2008). The concentration of total protein was determined using the 2-D Quant Kit (GE Healthcare, USA) according to manufacturer instructions. Equivalent amounts of total protein $(20 \mu \mathrm{g})$ were separated by $12.5 \%$ sodium dodecyl sulfate-polyacrylamide gel electrophoresis (SDS-PAGE) and transferred to nitrocellulose membranes using the iBlot Dry Blotting System (Invitrogen, USA) according to manufacturer instructions. Membranes were probed with the polyclonal antibody against the carboxyl region of soybean BiP (Figueiredo et al., 1997) at a 1:2000 dilution. As secondary antibody, the goat anti-rabbit IgG conjugated to alkaline phosphatase (Sigma, USA) was used. The 5-bromo-4-chloro-3-indolyl phosphate (BCIP) and $p$-nitroblue tetrazolium (NBT; Promega, USA) were used as substrates for the colorimetric reaction from alkaline phosphatase activity. BiP levels were quantified from membrane images using the GelQuant.Net 1.8.0 software (www.biochemlabsolutions.com) and the values of transgenic lines were calculated in relation to WT protein levels.

\section{Statistical analysis}

All measurements were repeated at least three times from different individual plants. Statistical differences between WT and transgenic lines were assessed based on analysis of variance (ANOVA) and differences in means were determined with the Student $t$-test, with critical values of $\mathrm{P} \leq 0.05$ and $\mathrm{P} \leq 0.01$.

\section{RESULTS}

\section{Overexpression of $\mathrm{EgPHI}-1$ enhances osmotic stress tolerance in transgenic tobacco}

We analyzed the effects of EgPHI-1 overexpression, at the whole plant level, on the response of plants to osmotic stress induced by $\mathrm{NaCl}$, mannitol, and $\mathrm{PEG}$. The transgenic lines showed increased tolerance to the different stresses compared with WT tobacco, even 25 days after the stress treatments (Figure 1). While the WT tobacco exhibited small leaves with chlorotic aspects and reduced growth in the $\mathrm{NaCl}$ treatment compared with the control treatment, growth of transgenic plants was not affected. Although PEG and mannitol treatments affected the growth of transgenic plants to some extent as compared with the control treatment, these effects were more severe in WT tobacco. PEG promoted slight chlorosis in the transgenic plants, while WT plants became completely necrotic under this treatment. Mannitol promoted 
a more severe chlorosis and reduced growth in the transgenic plants compared with the control treatment. It is interesting to note that under control conditions (without stress inductor), the transgenic plants exhibited higher growth than the WT plants.

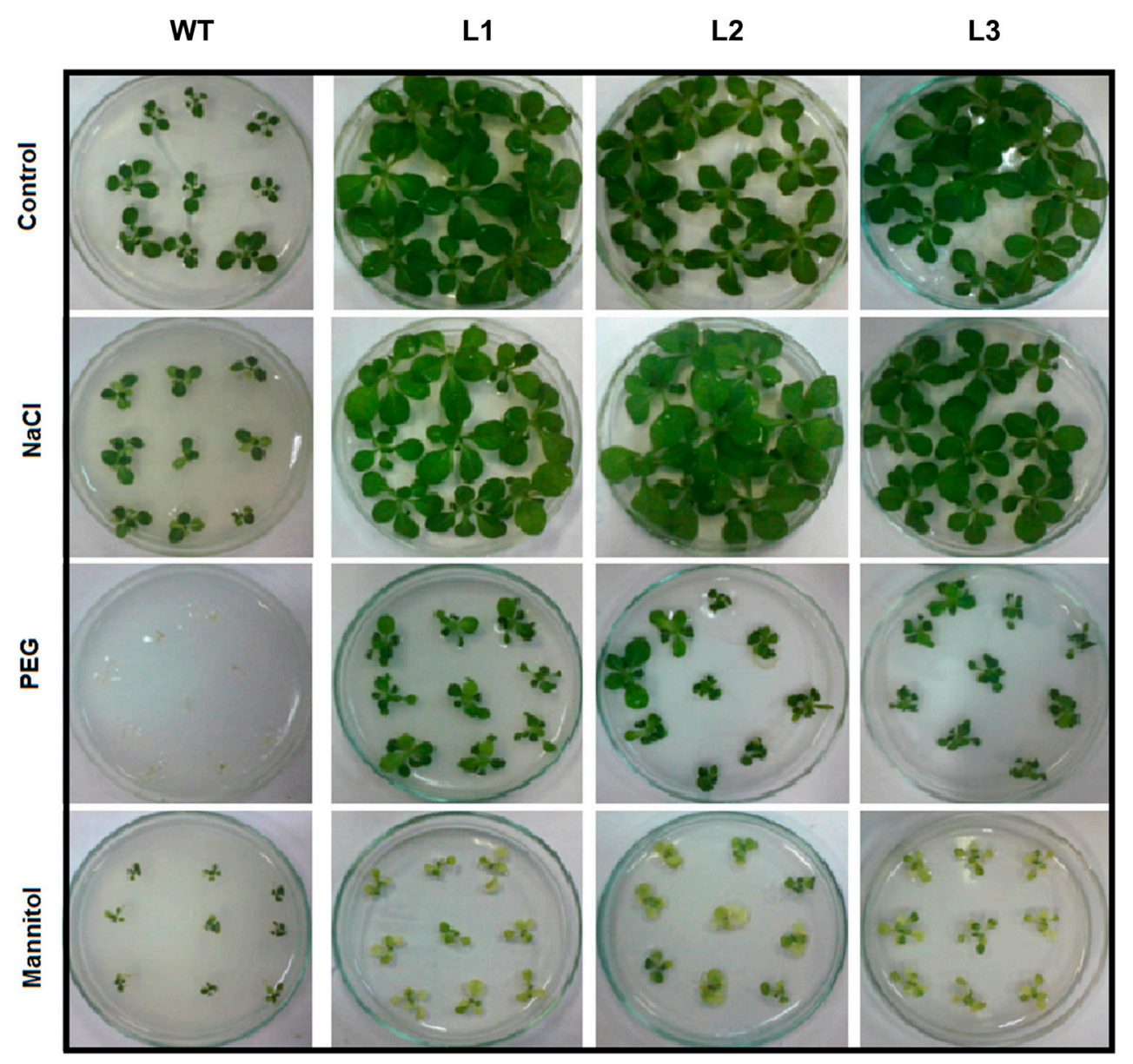

Figure 1. Phenotypes of tobacco plants exposed to different osmotic stress treatments. Fifteen-day-old wild-type (WT) and transgenic (L1, L2 and L3) tobacco plants were subjected to osmotic stress (-1.0 MPa) induced by $\mathrm{NaCl}$, polyethylene glycol (PEG) and mannitol. Plants were maintained in medium without stress inductors in the control treatment. Photographs were taken 25 days after the treatments.

A direct comparison between stressed WT and transgenic plants demonstrated significantly higher root length and biomass dry weight in the transgenic plants than in the WT plants under all stress conditions (Figure 2). The only exception was found in the mannitol treatment, in which the root length of transgenic plants was smaller than that of WT plants. However, the biomass dry weight of transgenic plants was $\sim 3$-fold higher than that of WT plants under this treatment. The transgenic lines, L1, L2, and L3, showed root lengths that were 3.7-, 4.7-, and 4.1-fold higher, respectively, than those of WT plants under $\mathrm{NaCl}$ treatment, whereas their 
biomass dry weights under this same treatment were, respectively, 3.7-, 6.4-, and 3.5-fold higher than WT plants. For the PEG treatment, the transgenic lines, L1, L2, and L3, showed a 123-, 142-, and 81-fold increase in root length, respectively, and a 7-, 12-, and 9-fold increase in biomass dry weight compared with WT plants.
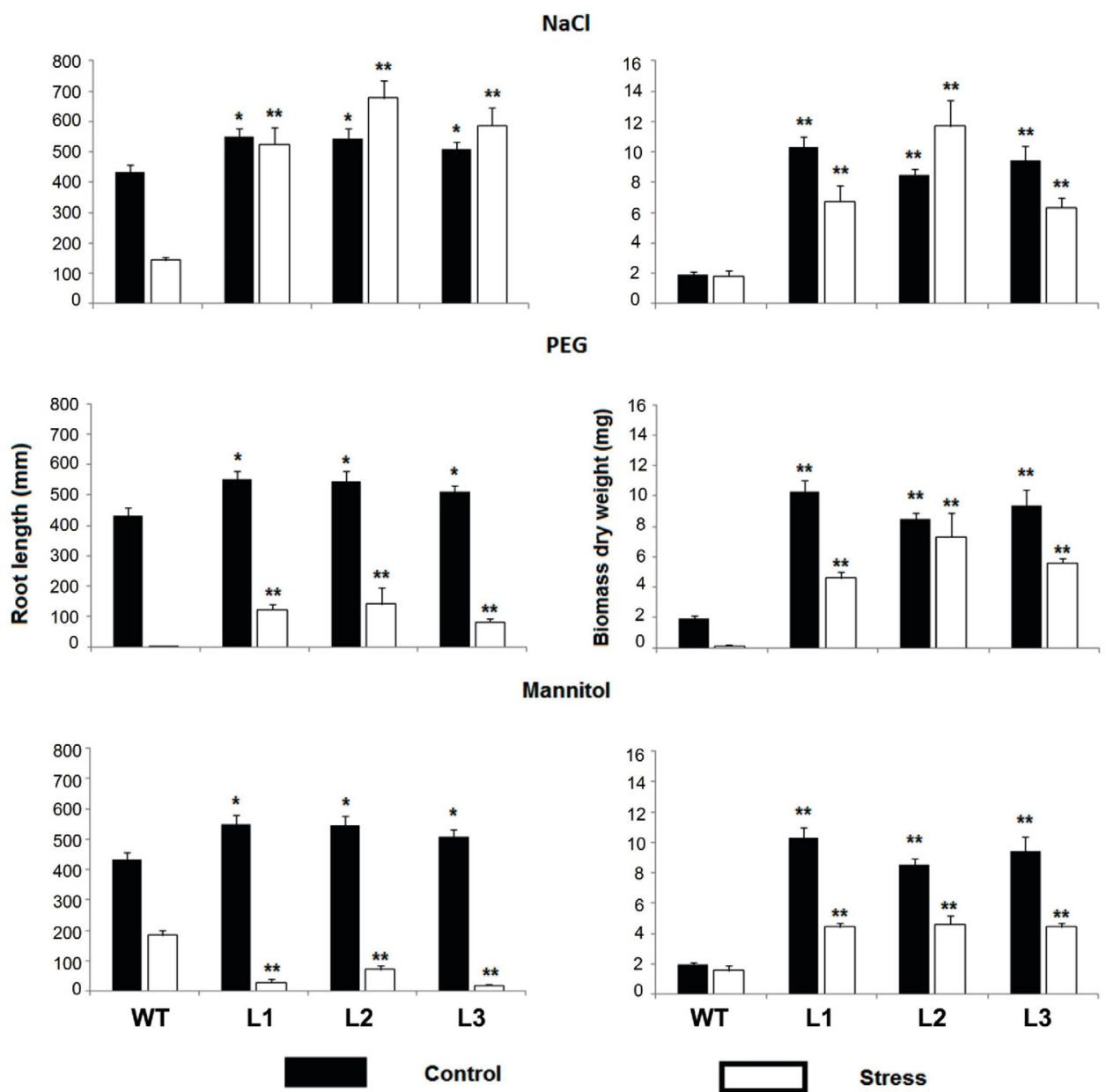

Figure 2. Root length (left) and biomass dry weight (right) of EgPHI-1 expressing transgenic tobacco plants under different stress treatments. Measurements were performed in wild-type (WT) and transgenic (L1, L2 and L3) plants subjected to osmotic stress induced by $\mathrm{NaCl}$, polyethylene glycol (PEG) and mannitol (open bars) or maintained under control (non-stress) conditions (filled bars), 25 days after the treatments. Bars represent the mean $\pm \mathrm{SE}$ of the three replicates $(\mathrm{N}=27)$. Statistically significant differences at $* \mathrm{P} \leq 0.05$ and $* * \mathrm{P} \leq 0.01$ between the WT and transgenic plants grown under control or stress conditions are indicated.

\section{EgPHI-1-mediated osmotic stress tolerance correlates with BiP expression}

The deduced amino acid sequence of EgPHI-1 is predicted to contain a signal 
peptide in its N-terminal portion targeting the ER (data not shown). Therefore, the constitutive overexpression of EgPHI-1 in the transgenic plants may disrupt ER homeostasis. This phenomenon usually results in the induction of ER-molecular chaperones, such as BiP, which has been demonstrated to mediate drought tolerance in transgenic plants (Alvim et al., 2001; Valente et al., 2009). To examine whether the observed osmotic stress tolerance of transgenic tobacco plants overexpressing EgPHI-1 correlates with $\mathrm{BiP}$ accumulation in plant tissues, $\mathrm{BiP}$ concentration was determined in leaves of WT and transgenic plants by Western blot analysis. All transgenic plants evaluated showed a remarkable increase in BiP accumulation as compared with WT tobacco (Figure 3), showing increases of 21, 45, and 85\% in L1, L2, and L3, respectively. Interestingly, the increase in BiP accumulation was proportional to the expression levels of EgPHI-1 in the transgenic lines. The transgenic line L3 was shown to accumulate the highest levels of EgPHI-1 transcripts, L2 had an intermediate level, and L1 showed the lowest transcript levels (Sousa AO, Assis ETCM, Silva DC, et al., unpublished results).

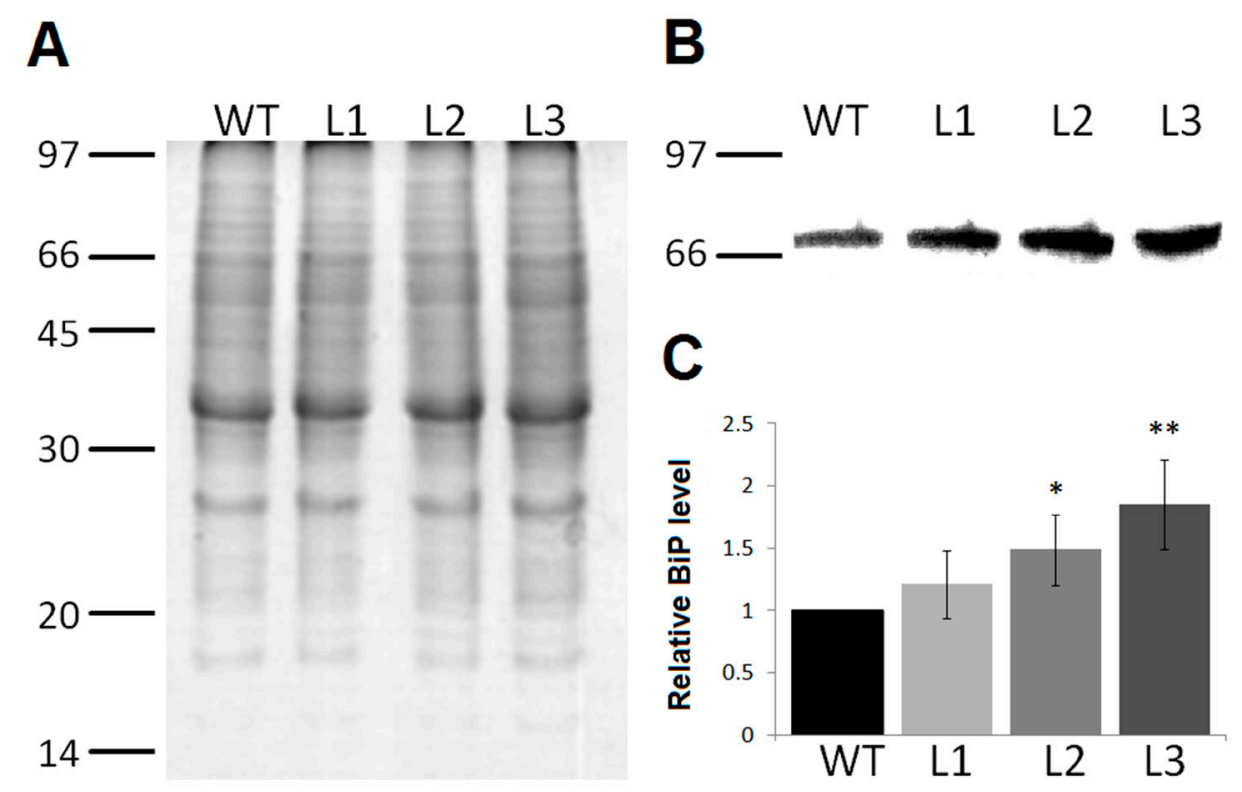

Figure 3. Binding protein (BiP) levels in wild-type (WT) and transgenic tobacco. A. Total protein extract. Equal amounts of total protein isolated from leaves of WT and transgenic (L1, L2 and L3) plants were separated by SDSPAGE. B. Western blot of BiP protein. Total protein extracts were immunoblotted with anticarboxyl BiP antibody. C. Relative BiP level. BiP levels were quantified from membrane images using the GelQuant.Net 1.8.0 software and the values of transgenic lines (L1, L2 and L3) were calculated in relation to WT protein levels. Bars represent the mean $\pm \mathrm{SE}$ of the three replicates $(\mathrm{N}=3)$. Statistically significant differences at $* \mathrm{P} \leq 0.05$ and $* * \mathrm{P} \leq 0.01$ between the WT and transgenic plants are indicated.

\section{DISCUSSION}

The results presented here show that EgPHI-1 expression modulates plant development and response to hyperosmotic conditions. The transgenic plants showed higher root length and biomass dry weight than the WT plants in control (non-stress) conditions. A similar 
phenotype was observed in transgenic Arabidopsis plants overexpressing EXO, another member of the PHI-1 family. EXO induced high growth of transgenic plants at the first stages of development by promoting the cellular expansion process (Coll-Garcia et al., 2004). Thus, our observations suggest a connection between $E X O$ and $E g P H I-1$ functions.

$\mathrm{NaCl}$, mannitol, and PEG induce hyperosmotic stress in different ways. $\mathrm{NaCl}$ penetrates through membranes and causes ionic stress and toxicity due to $\mathrm{Na}^{+}$excess. Mannitol also has the ability to penetrate through membranes, but it does not induce ionic stress, whereas PEG is a non-ionic and non-penetrating agent that decreases the water potential of the nutrient solution without causing toxicity (Gangopadhyay et al., 1997a,b). Therefore, the use of these three stress inducers allowed comparison of plant responses to osmotic stress by disturbance of water relationships, changes in the absorption and utilization of essential nutrients, and accumulation of toxic ions (Yokoi et al., 2002).

Transgenic tobacco plants expressing EgPHI-1 showed a significantly improved tolerance to osmotic stress induced by $\mathrm{NaCl}$, mannitol, and PEG as compared with WT plants. They displayed increased root lengths and biomass dry weights under all osmotic stress conditions, whereas WT tobacco showed progressive chlorosis, severe growth inhibition, and necrosis under the same stress conditions tested. The only exception was observed in the mannitol treatment, which inhibited the root length of transgenic plants relative to WT plants. This result suggests that EgPHI-1 might facilitate the penetration of mannitol through the root cell membranes and/or be involved in cellular processes in the root that are negatively affected by the presence of mannitol.

The tolerance of plants to salt stress has generally been attributed to their ability to accumulate $\mathrm{Na}^{+}$in intracellular compartments (He and Yu, 1995; Lutts et al., 1996). This mechanism allows better plant growth under salt stress (Thomas et al., 1992). The enhanced tolerance of EgPHI-1-expressing transgenic tobacco plants to $\mathrm{NaCl}$ treatment may be related to the increased intracellular compartmentalization of $\mathrm{Na}^{+}$or to the alleviation of intracellular $\mathrm{pH}$ changes caused by salt stress, as suggested for tobacco PHI-1 (Sano and Nagata, 2002). Tobacco PHI- 1 was postulated to play a role in alleviating changes of intracellular $\mathrm{pH}$ that are caused by stress, such as high concentrations of phosphate (Sano and Nagata, 2002), through its involvement in one of two possible mechanisms: i) intracellular proton exclusion from cells via an ATP-driven proton pump, and ii) proton consumption through $\mathrm{pH}$-sensitive decarboxylation reactions in organic acid metabolism (Sakano, 1998).

The most interesting observation of the present study was that BiP protein levels increased proportionally with EgPHI-1 expression levels in the transgenic lines. Thus, our results provide evidence that EgPHI-1 enhances osmotic stress tolerance through a mechanism that involves, at least partially, the modulation of $\mathrm{BiP}$ expression. $\mathrm{BiP}$ is an ER resident molecular chaperone that plays a central role in ER stress signaling by sensing alterations that affect protein folding and assembly in the ER environment (Malhotra and Kaufman, 2007). In addition to its role as an ER molecular chaperone, BiP overexpression in plants has also been shown to increase tolerance to drought stress (Alvim et al., 2001; Valente et al., 2009). BiP-mediated drought tolerance has not been associated with the typical short-term and longterm avoidance responses or with other well-known tolerance mechanisms (Valente et al., 2009), but has instead been associated with the prevention of stress-induced cell death by negatively regulating the stress-induced N-rich protein-mediated cell death response (Reis et al., 2011). The increased BiP levels in transgenic plants overexpressing EgPHI-1 may be 
a result of ER stress triggered by the input of EgPHI-1 protein in the ER. High protein input in the ER overloads the folding machinery and generates an elevation of unfolded proteins in the ER lumen, resulting in the induction of ER-molecular chaperones such as BiP (Malhotra and Kaufman, 2007). The induction of many chaperone and co-chaperone genes was observed in transgenic rice expressing $B i P 1$, and was associated with the ER stress response (Wakasa et al., 2011). These observations provide a fundamental basis for EgPHI-1-mediated osmotic stress tolerance that can be exploited for biotechnological applications in forestry, which can also be extended to other economically important crops.

\section{ACKNOWLEDGMENTS}

The authors are very grateful to Dr. Elizabeth P.B. Fontes from the Departamento de Bioquímica e Biologia Molecular, BIOAGRO, Universidade Federal de Viçosa, by providing the polyclonal antibody against the BiP protein. Research supported by Financiadora de Estudos e Projetos (FINEP, Brazilian Ministry of Science and Technology - MCT, grant \#2101063500), Conselho Nacional de Desenvolvimento Científico e Tecnológico (CNPq, MCT; grant \#50.6348/04-0, \#578632/08-0, \#311361/09-9), and Fundação de Amparo à Pesquisa do Estado da Bahia (FAPESB, Secretariat of Science, Technology and Innovation SECTI; grant \#BOL1569/2010).

\section{REFERENCES}

Alvim FC, Carolino SM, Cascardo JC, Nunes CC, et al. (2001). Enhanced accumulation of BiP in transgenic plants confers tolerance to water stress. Plant Physiol. 126: 1042-1054.

Bernard M (2003). Eucalyptus: A Strategic Forest Tree. In: Eucalyptus Plantations: Research, Management and Development (Wei R-P and Xu D, eds.). World Scientific, Singapure, 3-18.

Brinker M, Brosche M, Vinocur B, Abo-Ogiala A, et al. (2010). Linking the salt transcriptome with physiological responses of a salt-resistant Populus species as a strategy to identify genes important for stress acclimation. Plant Physiol. 154: 1697-1709.

Coll-Garcia D, Mazuch J, Altmann T and Mussig C (2004). Exordium regulates brassinosteroid-responsive genes. FEBS Lett. 563: 82-86.

Eldridge KG, Davidson J, Harwood CE and van Wyk G (1993). Eucalypt Domestication and Breeding. Clarendon Press Oxford, United Kingdom.

Figueiredo JEF, Cascardo JCM, Carolino SMB and Alvim FC (1997). Water-stress regulation and molecular analysis of the soybean BIP gene family. Braz. J. Plant Physiol. 9: 103-110.

Gangopadhyay G, Basu S and Gupta S (1997a). In vitro selection and physiological characterization of NaCl- and mannitol-adapted callus lines in Brassica juncea. Plant Cell Tissue Organ Cult. 50: 161-169.

Gangopadhyay G, Basu S, Mukherjee B and Gupta S (1997b). Effects of salt and osmotic shocks on unadapted and adapted callus lines of tobacco. Plant Cell Tissue Organ Cult. 49: 45-52.

He DY and Yu SW (1995). In vitro selection of a high-proline producing variant from rice callus and studies on its salt tolerance. Acta Phytophysiol. Sin. 21: 65-72.

Horsch RB, Fry JE, Hoffmann NL and Eichholtz D (1985). A simple and general method for transferring genes into plants. Science 227: 1229-1231.

Jefferson RA (1989). The GUS reporter gene system. Nature 342: 837-838.

Lutts S, Kinet JM and Bouharmont J (1996). Effects of salt stress on growth, mineral nutrition and proline accumulation in relation to osmotic adjustment in rice (Oryza sativa L.) cultivars differing in salinity resistance. Plant Growth Regul. 19: 207-218.

Mahajan S and Tuteja N (2005). Cold, salinity and drought stresses: an overview. Arch. Biochem. Biophys. 444: 139-158.

Malhotra JD and Kaufman RJ (2007). The endoplasmic reticulum and the unfolded protein response. Semin. Cell Dev. Biol. 18: 716-731. 
Murashige T and Skoog F (1962). A revised medium for rapid growth and bio assays with tobacco tissue cultures. Physiol. Plant. 15: 473-497.

Myburg AA, Potts BM, Marques CMP and Kirst M (2007). Eucalyptus. In: Genome Mapping and Molecular Breeding in Plants: Forest Trees (Kole CR, ed.). Springer, Berlin, 115-160.

Netting AG (2000). pH, abscisic acid and the integration of metabolism in plants under stressed and non-stressed conditions: cellular responses to stress and their implication for plant water relations. J. Exp. Bot. 51: 147-158.

Pasquali G, Bastolla FM, Pazzini F and Kirch RP (2005). Sequencing and Differential Expression of Xylem Specific Genes from two Eucalyptus Species with Highly Contrasting Wood Properties. Programme and Abstracts of the IUFRO Tree Biotechnology 2005, Pretoria, 22.

Pirovani CP, Carvalho HA, Machado RC, Gomes DS, et al. (2008). Protein extraction for proteome analysis from cacao leaves and meristems, organs infected by Moniliophthora perniciosa, the causal agent of the witches' broom disease. Electrophoresis 29: 2391-2401.

Reis PA, Rosado GL, Silva LA, Oliveira LC, et al. (2011). The binding protein BiP attenuates stress-induced cell death in soybean via modulation of the N-rich protein-mediated signaling pathway. Plant Physiol. 157: 1853-1865.

Sakano K (1998). Revision of biochemical pH-stat: involvement of alternative pathway metabolisms. Plant Cell Physiol. 39: 467-473.

Sano $\mathrm{T}$ and Nagata $\mathrm{T}$ (2002). The possible involvement of a phosphate-induced transcription factor encoded by phi-2 gene from tobacco in ABA-signaling pathways. Plant Cell Physiol. 43: 12-20.

Sano T, Kuraya Y, Amino S and Nagata T (1999). Phosphate as a limiting factor for the cell division of tobacco BY-2 cells. Plant Cell Physiol. 40: 1-8.

Thomas JC, De Armond RL and Bohnert HJ (1992). Influence of $\mathrm{NaCl}$ on growth, proline, and phosphoenolpyruvate carboxylase levels in Mesembryanthemum crystallinum suspension cultures. Plant Physiol. 98: 626-631.

Valente MA, Faria JA, Soares-Ramos JR, Reis PA, et al. (2009). The ER luminal binding protein (BiP) mediates an increase in drought tolerance in soybean and delays drought-induced leaf senescence in soybean and tobacco. J. Exp. Bot. 60: 533-546.

Vásquez-Robinet C, Watkinson JI, Sioson AA, Ramakrishnan N, et al. (2010). Differential expression of heat shock protein genes in preconditioning for photosynthetic acclimation in water-stressed loblolly pine. Plant Physiol. Biochem. 48: 256-264.

Wakasa Y, Yasuda H, Oono Y, Kawakatsu T, et al. (2011). Expression of ER quality control-related genes in response to changes in BiP1 levels in developing rice endosperm. Plant J. 65: 675-689.

Xiong L and Zhu JK (2002). Molecular and genetic aspects of plant responses to osmotic stress. Plant Cell Environ 25: 131-139.

Yokoi S, Bressan RA and Hasegawa PM (2002). Salt stress tolerance of plants. JIRCAS Working Rep. 25-33.

Zhu JK (2002). Salt and drought stress signal transduction in plants. Annu. Rev. Plant Biol. 53: 247-273. 